\title{
Blastomicosis norteamericana en un paciente diabético mal controlado, caso
}

\section{North American blastomicosis in a poorly controlled diabetic patient case report}

Lizbeth Karolina Vivanco Balcazar. ${ }^{1}$, Thalia Alexandra Feijóo Velepucha. ${ }^{2}$, Leonardo Santiago Sivisaka Satama. ${ }^{3}$ \& Ramiro Andrés Guerrero Iñiguez. ${ }^{4}$

\section{Recibido: 24-06-2019 / Revisado: 29-07-209 /Aceptado: 10-08-2019/ Publicado: 10-09-2019}

\begin{abstract}
DOI: https://doi.org/10.33262/cienciadigital.v3i3.4.834

Blastomycosis is a relatively rare and potentially lethal fungal infectious disease, which is mainly diagnosed in South Central and Midwest United States. Its presence in Latin America is exceptional. Pulmonary affection can vary from an asymptomatic infection to a severe and fatal disease, presented as acute or chronic pneumonia, or as a pulmonary mass.
\end{abstract}

We present the case of a patient who shown symptoms, such as a cough, mucopurulent expectoration, hemoptics, progressive dyspnea, unquantified fever and a weight loss over a period of three months, which despite the use of antibiotics did not remit. There are later complications with signs of septic shock, including respiratory difficulty with intercostal retractions, alteration of thoracic expandability and bilateral crackling of pulmonary fields.

On the radiograph, bilateral cottony infiltrates are observed and the study was complemented with culture, where the etiological microorganism could be visualized. The treatment with $200 \mathrm{mg}$ itraconazole is initiated, with favorable evolution of the patient, subsequently completely remitting the lung disease. On pulmonary pathology, a member of the Fungi group should be considered, focusing on the immunosuppressed patients to avoid future complications.

Keywords: Blastomycosis; Blastomyces dermatitidis; lung diseases, fungal.

\footnotetext{
${ }^{1}$ Hospital-Clínica San Agustín, Loja, Ecuador, karovi@ hotmail.es

${ }^{2}$ Hospital Eugenio Espejo, Quito, Ecuador, thaly.45@gmail.com

${ }^{3}$ Hospital Carlos Andrade Marín, Quito, Ecuador, santiago-santiagos@hotamil.com

${ }^{4}$ Hospital-Clínica San Agustín, Loja, Ecuador, raguerrero_93@hotmail.com
} 
ISSN: 2602-8085

\section{Resumen}

La blastomicosis es una enfermedad infecciosa micótica relativamente rara y potencialmente letal, que se diagnostica principalmente en el centro sur y medio oeste de Estados Unidos; siendo excepcional su presencia en Latinoamérica. La afectación pulmonar puede variar desde una infección asintomática hasta la enfermedad grave y mortal, presentándose como neumonía aguda o crónica, o bien como una masa pulmonar (Castillo CG, 2015)

Se presenta el caso de un paciente con el cuadro de tos, expectoración mucopurulenta, hemoptoica, disnea progresiva, fiebre no cuantificada y pérdida de peso de 3 meses de evolución que a pesar del uso de antibióticos no remite; complicándose posteriormente con signos de shock séptico abarcando dificultad respiratoria con retracciones intercostales, alteración de la expansibilidad torácica y crepitantes bilaterales de campos pulmonares, requiriendo el uso de inotrópicos. En la radiografía se observaron infiltrados algodonosos bilaterales y se complementó el estudio con cultivo, donde se pudo visualizar el microorganismo etiológico. Se inició el tratamiento con itraconazol de $200 \mathrm{mg}$, con evolución favorable del paciente, remitiendo posteriormente completamente la enfermedad pulmonar. Se deben considerar a pesar de no ser comunes en el medio, para patología pulmonar, los microorganismos fúngicos, haciendo un enfoque a los pacientes inmunodeprimidos para evitar las complicaciones a futuro.

Palabras claves: Blastomicosis, Blastomyces dermatitidis, enfermedades pulmonares, fúngica.

\section{INTRODUCCIÓN}

La blastomicosis es una infección micótica endémica, grave, poco frecuente, causada por el hongo dimórfico, Blastomyces dermatitidis. La presentación inicial se produce a través de los pulmones y suele ser subclínica. Se caracteriza por ser una enfermedad progresiva que implica múltiples órganos y sistemas y en el peor de los casos una infección pulmonar aguda fulminante. (Bradsher A. R., 2016)

El microorganismo responsable habita generalmente en climas templados húmedos, con suelos ricos y ácidos, relacionado con la madera en descomposición, y se encuentra en las cercanías de los ríos o lagos o en las zonas sujetas a inundaciones periódicas. Es propia de los trabajadores rurales; agricultores y campesinos y la vía de entrada más frecuente es a través de inhalación de esporas. (Marek A. Martynowicz, 2012)

Epidemiológicamente la blastomicosis es endémica en el continente norteamericano. Se extiende desde Canadá, Estados Unido y México. A nivel mundial la incidencia varía según 
las áreas afectadas, siendo alta en las áreas endémicas. La incidencia es menos de 1 caso por 100,000 habitantes norteamericanos. A nivel local los reportes de esta patología son relativamente escasos. (Bradsher A. R., 2016)

Es frecuente a los 30 y 50 años (promedio cuarta década). Los hombres adultos tienen la mayor probabilidad de desarrollar la patología. (Dennis J. Baumgardner, 2014)

El cuadro se caracteriza por un mal estado general, anorexia, pérdida de peso, sudores nocturnos, disnea, tos mucopurulenta o hemoptisis y dolor torácico. En el estudio radiológico se comprueban infiltrados de diversos tamaños, por lo general densos y parahiliares. (George A. Sarosi, 2013)

En ocasiones, su diagnóstico es tardío precisamente por sus características clínicas y radiológicas que muchas veces inducen al diagnóstico erróneo de neumonía bacteriana y viral. (George A. Sarosi, 2013)

Hay que tener en cuenta que la enfermedad pulmonar tiende a ser más grave en huéspedes inmunocomprometidos ya que el curso clínico es más agresivo, y hay una tasa de mortalidad más alta. (Marek A. Martynowicz, 2012)

El diagnóstico se basa fundamentalmente en la clínica, el tratamiento tórpido a los antibióticos y especialmente al hallazgo microscópico y el aislamiento en cultivos de B. Dermatitidis en muestras de esputo, lavados bronquiales, pus, biopsias, y rara vez hemocultivos. Las opciones de tratamiento para los pacientes con blastomicosis incluyen anfotericina B o uno de los azoles por lo general itraconazol. (Bradsher R. W., 2015)

La importancia radica que, al tratarse de una afección pulmonar relativamente rara y potencialmente letal, se debe ampliar el conocimiento de la presentación de esta patología especialmente en pacientes inmunocomprometidos con el fin de plantear un diagnóstico precoz y prevenir complicaciones severas. (Dennis J. Baumgardner, 2014)

Se presenta un caso de en un paciente de sexo masculino inmunodeprimido (Diabetes Mellitus tipo II mal controlada) con manifestaciones características de un cuadro de Neumonía, en el cual se aísla B. Dermatitidis.

\section{PRESENTACIÓN DEL CASO CLÍNICO}

Paciente masculino de 53 años de edad residente del Cantón Calvas de la provincia de Loja. Con antecedentes de Diabetes Mellitus tipo II de 10 años de evolución, patología mal controlada. Dedicado a la agricultura y albañilería.

Refirió antecedente de pérdida de peso de 3 meses de evolución que se acompañó de tos con expectoración mucopurulenta, hemoptoica. Posteriormente presentó disnea progresiva de grandes a pequeños esfuerzos, además 9 días previo al ingreso presenta fiebre no cuantificada, motivo por el cual acude al hospital Isidro Ayora de la Cuidad de Loja con el diagnóstico presuntivo de diabetes descompensada, neumonía grave adquirida en la comunidad y shock séptico de foco pulmonar. Recibió soporte con inotrópicos.

Al examen físico de ingreso: caquéctico, diaforética piel caliente, mucosas pálidas y secas, Frecuencia respiratoria: $37 \mathrm{rpm}$, Frecuencia cardíaca: $130 \mathrm{lpm}$, Temperatura: 38,4, Saturación de oxígeno: $84 \%$, Tensión arterial: $85 / 55 \mathrm{mmHg}{ }^{\circ} \mathrm{C}$, tos con abundante expectoración herrumborsa, dificultad respiratoria marcada al reposo, tiraje intercostal, 
retracciones intercostales, alteración de la expansibilidad torácica, crepitantes bilaterales en ambos campos pulmonares, e hipoventilación. Glasgow:14/15

Se decidió ingreso a críticos en virtud del cuadro clínico que cursa y los siguientes datos de laboratorio: Glucemia: $299 \mathrm{mg} / \mathrm{dl}$, Neutrófilos: 85,2 K/uL VES: 25mm/Hg, Gasometría: PCo2: $13.10 \mathrm{mmHg}$, Ph: 7.41, Sat:94.4\% (Alcalosis respiratoria), Rx simple de tórax: Infiltrados algodonosos bilaterales (Ver imagen 1), TAC simple tórax: infiltrado mixto en vidrio delustrado (Ver imagen 2) Índice SOFA: 5 APACHE: 12 Índice de mortalidad: 15\%. Se plantea el uso de Ventilación asistida.

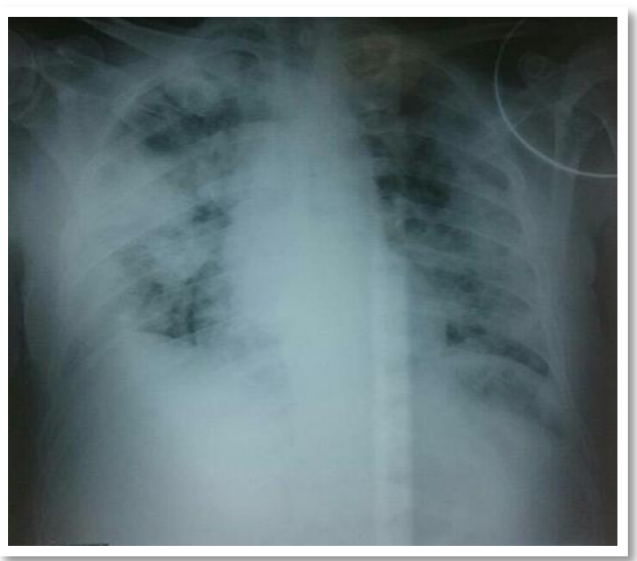

Imagen 1. Radiografía simple de Tórax

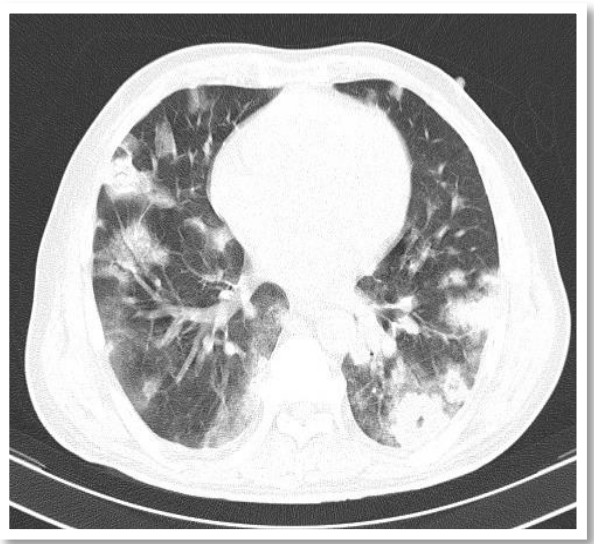

Imagen 2. TAC simple de Tórax

Posteriormente debido al aspecto herrumbroso del esputo (Ver imagen 3) se realiza examen directo de la muestra y se evidencia la presencia hifas tabicadas con microconidias y levaduras +++

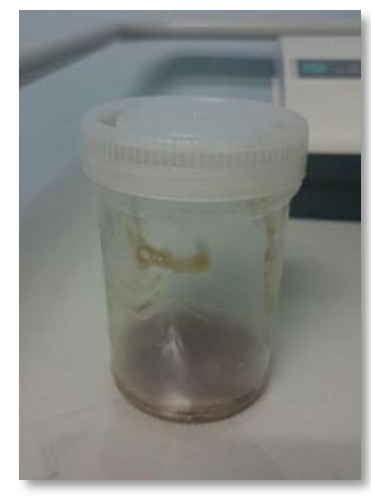

Imagen 3. Características del Esputo

El resultado del cultivo mostró abundantes blastosporas redondeadas de gran tamaño con aspecto similar a un hongo dimórfico, el mismo que requirió de una temperatura de $37^{\circ}$ para su aislamiento óptimo. Compatible con Blastomyces dermatitis. 

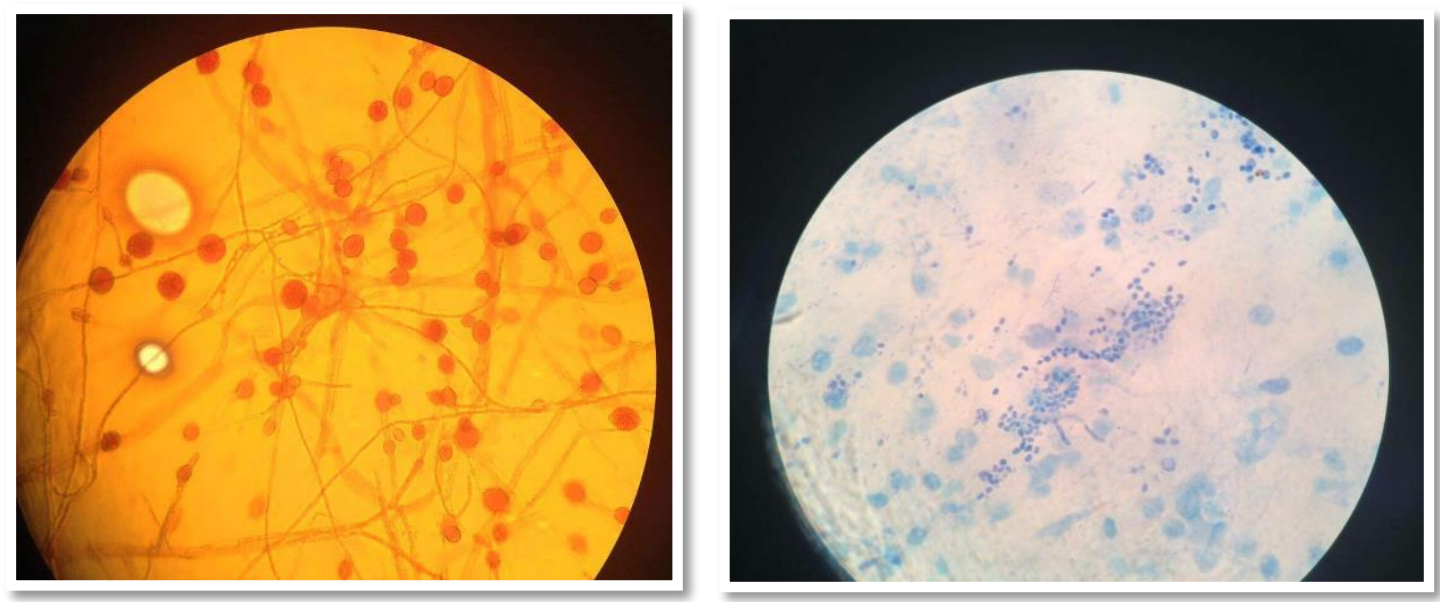

Imagen 4 y 5 . Estudio microscópico de la muestra

Debido a los resultados característicos se planteó el diagnóstico de Blastomicosis Pulmonar y se inició tratamiento antifúngico con Itraconazol de $200 \mathrm{mg}$ vía oral cada 12 horas durante 6 meses. Evolucionó favorablemente. Así mismo se realizó controles seriados de la función suprarrenal ya que las dosis elevadas de este agente terapéutico pueden causar daños colaterales.

Luego de tres meses el paciente evoluciona clínicamente favorable y la placa de control muestra cambios significativos.

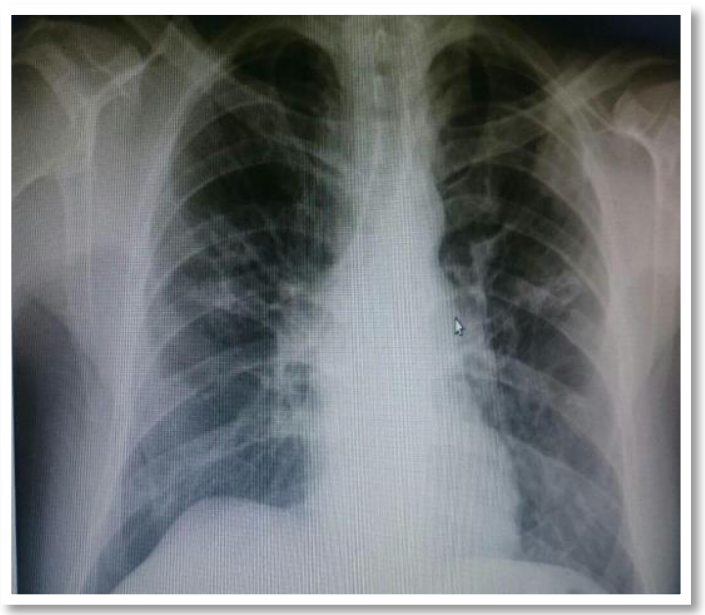

Imagen 6. Rx de tórax de control

\section{DISCUSIÓN}

La blastomicosis es una infección causada por el hongo dimórfico Blastomyces dermatitidis, considerada endémica en áreas de América del Norte, aunque se ha distribuido ampliamente en el continente africano y en regiones del Medio Oriente y la India (Brown, y otros, 2018). 
Casos de pacientes infectados por $B$. dermatitidis, sin antecedentes de viajes a zonas endémicas, que hayan sido reportados en América Latina son excepcionales. En Ecuador no existe ningún caso reportado previamente sobre esta patología, por lo que podríamos estar frente al primer reporte de infección por Blastomyces dermatitidis en nuestro país.

Se cree que el mecanismo causal inicial de la infección pulmonar es la inhalación de los conidios de B. dermatitidis. En la naturaleza, el hongo existe en forma micelial y la inhalación de esporas da como resultado una infección pulmonar primaria. Las esporas se inoculan en las células alveolares y se produce la germinación en formas de levadura. Aunque los macrófagos pulmonares eliminan la mayoría de las esporas antes de que ocurra la infección, las que sobreviven producen neumonitis. Desde el pulmón, puede haber diseminación hematógena y, con menos frecuencia, diseminación linfática. La respuesta inmune a la infección consiste en la migración de macrófagos neutrófilos hacia el tejido infectado, lo que induce una respuesta granulomatosa y supurativa con necrosis y fibrosis asociadas a la blastomicosis (Bradsher R. J., 2008). El germen se encuentra típicamente en suelos cálidos, húmedos y ricos en desechos orgánicos; por lo tanto, el mayor riesgo de infección es la exposición al suelo cerca de vías fluviales o en áreas boscosas. Sin embargo, existe evidencia de que algunos casos pueden surgir en el hogar, especialmente en sótanos y áticos (Baumgardner \& Paretsky, 2001). Si bien las medidas preventivas generales son difíciles, las personas con sistemas inmunes debilitados tienen un mayor riesgo de blastomicosis. Nuestro paciente no presentó antecedente de viaje a zona endémica, ni había visitado áreas próximas a vías fluviales, sin embargo, habita en una zona rural del Ecuador y presenta inmunosupresión debido a al mal control de la diabetes mellitus.

$B$ dermatitidis puede producir una variedad de síndromes clínicos, desde una infección asintomática hasta el síndrome de dificultad respiratoria aguda fulminante (Davies \& Sarosi, 1997), es por ende que se requiere un alto grado de sospecha para su diagnóstico. Los pulmones sirven como portal de entrada. La infección pulmonar puede manifestarse como neumonía aguda o crónica, siendo esta última más común (Bradsher R. J., 2008). La capacidad de imitar otras enfermedades es un sello distintivo de la blastomicosis, y su similitud con neumonías por otro agende causal y con la tuberculosis pulmonar a menudo conduce a errores o retraso en el tratamiento. Los síntomas clínicos generalmente incluyen tos, fiebre, pérdida de peso y dolor en el pecho (Saccente \& Woods, 2010). En algunos casos puede presentarse hemoptisis (Fang, Washington, \& Kumar, Imaging manifestations of blastomycosis: a pulmonary infection with potential dissemination, 2007). En correlación, nuestro paciente un síndrome de dificultad respiratoria aguda, fiebre, pérdida de peso y hemoptisis. 
Las manifestaciones radiológicas de la infección pulmonar son muy diversas y principalmente incluyen consolidación del espacio aéreo, lesiones similares a masas, infiltración intersticial y miliar, y lesiones cavitarias. La consolidación es el hallazgo radiográfico más común y a menudo se confunde con una neumonía bacteriana. Las imágenes sugestivas de masas en el tórax constituyen el segundo hallazgo radiográfico más común, y se han se han reportado hasta en un $31 \%$ de los casos; típicamente en pacientes con una presentación crónica de la enfermedad (Fang, Washington, \& Kumar, Imaging manifestations of blastomycosis: a pulmonary infection with potential dissemination, 2007). En nuestro informe se pudo evidenciar infiltrados algodonosos bilaterales en la Rx de tórax y un patrón de vidrio delustrado en la imagen de TAC.

Para establecer un diagnóstico definitivo de blastomicosis pulmonar, $B$. dermatitidis debe aislarse en un cultivo de esputo o en un espécimen de aspirado bronquial, u observarse en el examen histológico del tejido obtenido por biopsia (bronquio o pulmón). Aunque el rendimiento diagnóstico de las preparaciones húmedas del esputo es bajo, debe considerarse dado el bajo costo y la simplicidad. El diagnóstico se realiza mediante la observación de la característica levadura incipiente de paredes gruesas y de base amplia después de tinciones especiales (Martynowicz \& Prakash, 2002). El germen puede ser observado en el cultivo de agar dextrosa de Sabouraud a temperatura ambiente como un moho blanco esponjoso. Nuestro diagnóstico se sustentó en el examen microbiológico, donde se visualizaron células típicas con morfología propia de Blastomices rodeadas de una reacción inflamatoria con neutrófilos y linfocitos.

La resolución espontánea de la blastomicosis no parece ocurrir y la enfermedad no tratada puede asociarse con tasas de mortalidad cercanas al 60\% (Saccente \& Woods, 2010). El tratamiento de la blastomicosis se ha ampliado con la disponibilidad de los antifúngicos azólicos, a saber, ketoconazol, itraconazol y fluconazol. Hasta la fecha, sin embargo, no se han realizado estudios cegados aleatorios que comparen diferentes regímenes. Las recomendaciones de tratamiento publicadas para la blastomicosis se basan en ensayos controlados relativamente pequeños, series de casos y experiencia anecdótica. El itraconazol, en dosis que oscilan entre 200 y $400 \mathrm{mg}$ diarios, se considera el fármaco de elección para pacientes con blastomicosis no mortal que no pone en peligro el SNC porque está asociado con una mejor tolerancia y eficacia, con menos toxicidad que el ketoconazol (Proia \& Harnisch, 2012). Para los pacientes con enfermedad grave, potencialmente mortal o del SNC, el desoxicolato de anfotericina B sigue siendo el fármaco de elección (McBride, Gauthier, \& Klein, 2017). En correlación nuestro paciente tuvo una excelente evolución clínica, en base al tratamiento instaurado con itraconazol.

\section{CONCLUSIONES}

- Se presentó un caso de un paciente masculino de 53 años agricultor que inicialmente cursa con clínica compatible de neumonía adquirida en la comunidad, descompensada metabólicamente por una diabetes mellitus tipo II mal controlada. 
Recibió tratamiento con antimicrobiano de amplio espectro cuya evolución no fue satisfactoria. Por lo que se adicionó empíricamente anti fúngico dada a la presentación clínicas y a las características macroscópicas del esputo. Una vez realizados los exámenes pertinentes (examen directo y cultivo) de donde resulto el aislamiento de B. Dermatitis, se diagnosticó una micosis profunda de origen pulmonar.

- Es importante que, ante un paciente con los factores de riesgo epidemiológico aunado a la presentación clínica tórpida y rápidamente progresiva, se debe tomar en cuenta otras etiologías de neumonías adquirida en la comunidad como las de origen micótico.

- Por tal motivo es muy relevante para el medico conocer que, ante la evolución desfavorable, o de una infección respiratoria baja, en un paciente con los factores de riesgo ya descritos debe tomarse en cuenta la etiología micótica tal como lo señala la literatura. (Schwartz, Embil, Sharma, Goulet, \&amp; Bruce, 2016)

\section{Referencias}

Wulff-Pérez, M., Martín-Rodríguez, A., Gálvez-Ruiz, M., \& de Vicente, J. (2013). The effect of polymer surfactant on the rheological properties of nanoemulsions. Colloid and Polymer Science, 291, 709-716.

Zamora Mora, V., Soares, P., Echeverria, C., Hernández , R., \& Mijangos, C. (2015). Composite chitosan/Agarose ferrogels for potential applications in magnetic hyperethermia. Gels. , 1, 69-80.

Bhat, S., Tripathi, A., \& Kumar, A. (2010). Supermacroprous chitosan-agarose-gelatin cryogels. in vitro characterization and in vivo assesment for cartilage tissue engineering. Journal of the Royal Society Interface, 1-15.

Ruiz Estrada, G. (2004). Desarrollo de un Sistema de liberación de fármacos basado en nanopartículas magnéticas recubiertas con Polietilénglicol para el tratamiento de diferentes enfermedades. Madrid: Universidad Autónoma de Madrid. Departamento de Física Aplicada.

Cortés, J., Puig, J., Morales, J., \& Mendizábal, E. (2011). Hidrogeles nanoestructurados termosensibles sintetizados mediante polimerización en micro emulsión inversa. Revista Mexicana de Ingeniería Química., 10 (3), 513-520.

Song , J., King, S., Yoon , S., Cho, D., \& Jeong, Y. (2014). Enhanced spinnability of narbon nanotube fibers by surfactant addition. Fiberes and Polymers , 15 (4), 762-766.

Ilg, P. (2013). Stimuli-responsive hydrogels cross-linked by magnetic nanoparticles. Soft Matter , 9, 3465-3468. 
Bossis, G., Marins, J., Kuzhir, P., Volkova, O., \& Zubarev, A. (2015). Functionalized microfibers for field-responsive materials and biological applications. Journal of Intelligent Material Systems and Structures, 1-9.

Lin, Y.-S., Huang, K.-S., Yang, C.-H., Wang, C.-Y., Yang, Y.-S., Hsu, H.-C., y otros. (2012). Microfluidic synthesis of microfibers for magnetic-responsive controlled drug release and cell culture. PLoS ONE, 7 (3), 1-8.

Tartaj, P., Morales, M., González-Carreño, T., Veintemillas-Verdaguer, S., \& Serna, C. (2005). Advances in magnetic nanoparticles for biotechnology applications. Journal of Magnetism and Magnetic Materials , 290, 28-34.

García-Cerda, L., Rodríguez-Fernández, O., Betancourt-Galindo, R., Saldívar-Guerrero, R., \& Torres-Torres, M. (2003). Síntesis y propiedades de ferrofluidos de magnetita. Superficies y Vacío. , 16 (1), 28-31.

Dias, A., Hussain, A., Marcos, A., \& Roque, A. (2011). A biotechnological perspective on the application of iron oxide magnetic colloids modified with polysaccharides. Biotechnology Advances 29 , 29, 142-155.

Lewitus, D., Branch, J., Smith, K., Callegari, G., Kohn, J., \& Neimark, A. (2011). Biohybrid carbon nanotube/agarose fibers for neural tissue engineering. Advanced Functional Materials , 21, 2624-2632.

Estrada Guerrero, R., Lemus Torres, D., Mendoza Anaya, D., \& Rodriguez Lugo, V. (2010). Hidrogeles poliméricos potencialmente aplicables en Agricultura. Revista Iberoamericana de Polímeros , 12 (2), 76-87.

Aldana, S., Vereda, F., Hidalgo-Alvarez, R., \& de Vicente, J. (2016). Facile synthesis of magnetic agarose microfibers by directed selfassembly. Polymer , 93, 61-64.

Castillo CG, K. C. (Diciembre de 2015). Blastomicosis. ELSERVIER , 1.

Brown, E., McTaggart, L., Dunn, D., Pszczolko, E., Tsui, K., Morris, S., y otros. (2018). Epidemiology and Geographic Distribution of Blastomycosis, Histoplasmosis, and Coccidioidomycosis, Ontario, Canada, 1990-2015. Emerg Infect Dis , 24 (7), 12571266.

Baumgardner, D., \& Paretsky, D. (2001). Blastomycosis: more evidence for exposure near one's domicile. $W M J, 100(7), 43-45$. 
Bradsher, R. J. (2008). Pulmonary Blastomycosis. Semin Respir Crit Care Med, 29 (2), 174 181.

Saccente, M., \& Woods, G. (2010). Clinical and laboratory update on blastomycosis. Clin Microbiol Rev , 23 (2), 367-381.

Davies, S., \& Sarosi, G. (1997). Epidemiological and clinical features of pulmonary blastomycosis. Semin Respir Infect. 1997, 12, 206-18.

Fang, W., Washington, L., \& Kumar, N. (2007). Imaging manifestations of blastomycosis: a pulmonary infection with potential dissemination. Radiographics , 27 (3), 641-55.

Fang, W., Washington, L., \& Kumar, N. (2007). Imaging manifestations of blastomycosis: a pulmonary infection with potential dissemination. Radiographics , 27 (3), 641-655.

Martynowicz, M., \& Prakash, U. (2002). Pulmonary blastomycosis: an appraisal of diagnostic techniques. Chest , 121 (3), 768-773.

Proia, L., \& Harnisch, D. (2012). Successful use of posaconazole for treatment of blastomycosis. Antimicrob Agents Chemother , 56 (7), 4029.

McBride, J., Gauthier, G., \& Klein, B. (2017). Clinical manifestations and treatment of blastomycosis. Clin Chest Med , 38 (3), 435-449.

Bradsher, A. R. (Mayo de 2016). Clinical manifestations and diagnosis of blastomycosis. UptoDate, 2.

Marek A. Martynowicz, a. U. (2012). Pulmonary Blastomycosis. An Appraisal of Diagnostic Techniques. American College of Chest Physicians , 3.

Dennis J. Baumgardner, J. L. (Abril de 2014). The Differential Diagnosis of Pulmonary Blastomycosis Using Case Vignettes: A Wisconsin Network for Health Research (WiNHR) Study. WMJ .

George A. Sarosi, K. J. (2013). Clinical Features of acute Pulmonary Blastomicosis. The new England Journal of Medicine, 2.

Bradsher, R. W. (Junio de 2015). Treatment of blastomycosis. UptoDate , 2.

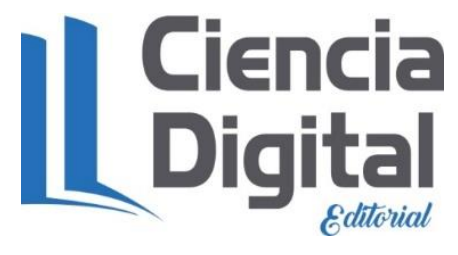


PARA CITAR EL ARTÍCULO INDEXADO.

Vivanco Balcazar, L., Feijóo Velepucha, T., Sivisaka Satama, L., \& Guerrero Iñiguez, R. (2019). Blastomicosis norteamericana en un paciente diabético mal controlado, caso. Ciencia Digital, 3(3.4.), 49-59. https://doi.org/10.33262/cienciadigital.v3i3.4.834

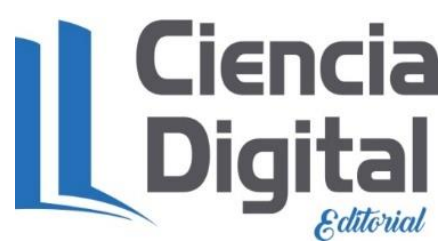

El artículo que se publica es de exclusiva responsabilidad de los autores y no necesariamente reflejan el pensamiento de la Revista Ciencia Digital.

El artículo queda en propiedad de la revista y, por tanto, su publicación parcial y/o total en otro medio tiene que ser autorizado por el director de la Revista Ciencia Digital.
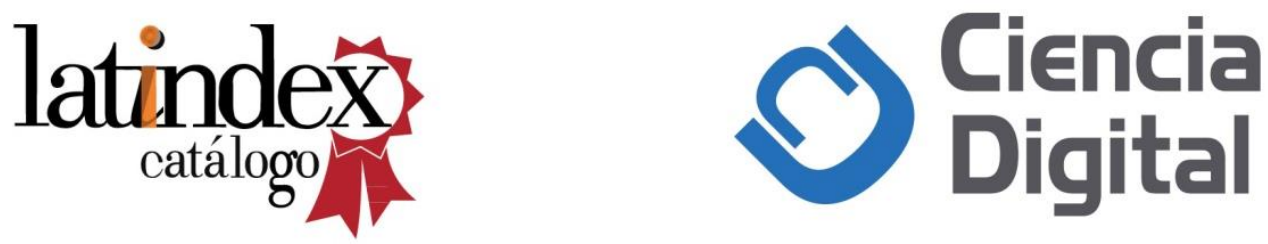